\title{
MEDIENWISSENSCHAFT DER MOTIVE
}

Unser folgender Beitrag schlägt ein großes filmwissenschaftliches Forschungsprojekt vor. Dieses Projekt einer kinematografischen Motivforschung nimmt einerseits Forschungen auf, wie sie schon früher immer wieder einmal unternommen worden sind. Es setzt aber erstens ein mit einer Reflexion auf Funktion und Leistung des kinematografischen Motivs und versieht sie so mit einem neuen Grundkonzept. Zudem schlägt es vor, der kinematografischen Motivforschung ein neues Werkzeug zur Verfügung zu stellen, das eine neue, verteilte Arbeitsweise ermöglichen soll: eine Motivdatenbank. Drittens bemüht das Projekt sich darum, der Filmwissenschaft Perspektiven im Hinblick auf eine umfassendere Medienwissenschaft zu eröffnen - und umgekehrt.

Wir beginnen zunächst mit einer Vorüberlegung zu diesem letzten Punkt. Für uns zeichnet Medienwissenschaft sich unter anderem dadurch aus, dass sie an einem dezidierten Interesse am Materiellen festhält, an der dinglich verfassten Basis aller Praxis der Sinn- und Wissensproduktion, an technischen und ästhetischen Artefakten beispielsweise. Sie untersucht die Mitwirkung und Mitarbeit der Dinge an unseren Handlungen und Erkenntnissen. Sie ist aber nicht der einzige Ort, an dem solches Spezialinteresse an den Dingen ausgebildet und prozessiert wird. Zum Beispiel wird in einer bemerkenswerten Tradition der Film als ein Ort begriffen, an dem die Dinge in besonderer Weise in den Focus gerückt werden. Unsere These dazu geht - z. B. mit Siegfried Kracauer - davon aus, dass der Film ein audiovisuelles und daher stets aufs Sinnliche und Konkrete, Einzelne und Materielle gehendes Medium ist. ${ }^{1}$ Hier häuft er sein spezifisches Wissen an und

1 Siegfried Kracauer, Theorie des Films, Frankfurt/M. (Suhrkamp) 1979. bringt es zur Geltung. Und Geltung verschafft der Film den Dingen, indem er ihnen Operationsmacht im kinematografischen Zusammenhang zuweist. Solche im 
Zusammenhang wirksamen, operativen Objekte wollen wir als kinematografische Motive erforschen. Denn es scheint uns, als könnte der Film ein besonders interessantes medienwissenschaftliches Beobachtungsobjekt sein, das immer schon das leistet, worum Medienwissenschaft auf ihre Weise sich ebenfalls bemüht. Sie könnte dann vom Film lernen oder sich ihm gegenüber als Beobachtung zweiter Ordnung etablieren.

\section{Die Jalousie}

Mühelos können zahlreiche Beispiele für kinematografische Motive im Sinne der hier vorgeschlagenen Forschungen angeführt werden. $\mathrm{Zu}$ vielen liegen auch bereits einschlägige Untersuchungen vor, ohne dass jedoch daraus eine systematische oder historische Motivik entwickelt worden oder auch nur ein begründeter filmspezifischer Motivbegriff entfaltet worden wäre. ${ }^{2}$ Hier wollen wir zur Verdeutlichung einmal ansatzweise auf ein paradigmatisches Motiv eingehen, nämlich auf die Jalousie. ${ }^{3}$

Die Jalousie ist ein Requisit oder ein Teil des Szenenbildes, ein sichtbares und wohldefiniertes Objekt des Films. Und als Requisit oder Teil des Szenenbildes besitzt sie zunächst keine bedeutungstragende, semantische oder ikonografische Dimension oder Tradition. Sie ist als Motiv vollkommen funktional. Sie macht sichtbar und unsichtbar, schließt ein und aus, aber sie verweist auf nichts außer eben auf das, was sie tut. Gerade darin aber gibt die Jalousie erstens ein bestimmtes Gepräge, das sie einem Bild, einer Einstellung, einer Sequenz, einem Ort im Film, bisweilen einem ganzen Film verleiht - eine bestimmte Atmosphäre etwa. Die Jalousie kann also, wie im Film Noir, ein stilbildendes oder zumindest stilaffines Motiv sein. Entscheidend dabei ist, dass sie zugleich Ursache des bildnerischen Stileffekts - also hier: der typischen gerasterten Hell-Dunkel-Lichtführung des Noir - und sichtbares Objekt innerhalb des Films selbst ist. Als sichtbares Objekt im Bild motiviert das Motiv der Jalousie zugleich die Lichtführung, unter die das ganze Bild gesetzt wird. Die handelnden Figuren des Films bewegen sich dann im Jalousienlicht, in das sie gesetzt werden und das zur Bedingung ihres Erscheinens und ihrer Sichtbarkeit wird. Eines der prägnantesten Beispiele dafür mag die Wohnzimmersequenz aus Billy Wilders Double Indemnity sein. Hier ist auch besonders schön der zweideutige, vermittelnde Charakter des Motivs zu erkennen: das zweidimensionale Lichtraster, das über die dreidimensionalen Körper gelegt wird, akzentuiert sie in den Deflektionen des Schattenwurfs als Oberflächen. Es vermittelt so zwischen der Zweidimensionalität, die das Bild selbst ausmacht, mit der Dreidimensionalität der handelnden Figuren und der diegetischen Welt. Die Jalousie lässt dabei an ihrer - zunächst ästhetischen - Wirksamkeit, an ihrem Charakter als Ursache, bewirkendes (und bewirktes) Objekt, als 〈Index> (im Sinne der anthropologischen Ästhetik Alfred Gells ${ }^{4}$ ) keinen Zweifel zu.
2 Forschergruppe Telefonkommunikation (Hg.), B. Debatin (Bearb.), H. J. Wulff (Bearb.), Telefon und Kultur. Das Telefon im Spielfilm, Berlin (Spiess) 1991.

3 Hier greifen wir auf frühere Forschungen zurück, ausführlicher dargestellt in: Lorenz Engell, Die Jalousie. Ein Muster der Lichtführung und der Aufklärung, in: ders., Ausfahrt nach Babylon. Vorträge und Essays zur Kritik der Medienkultur, Weimar (Verlag und Datenbank für Geisteswissenschaften) 2000, 325-340.

4 Alfred Gell, Art and Agency. An Anthropological Theory, Oxford (Oxford University Press) 1998. 


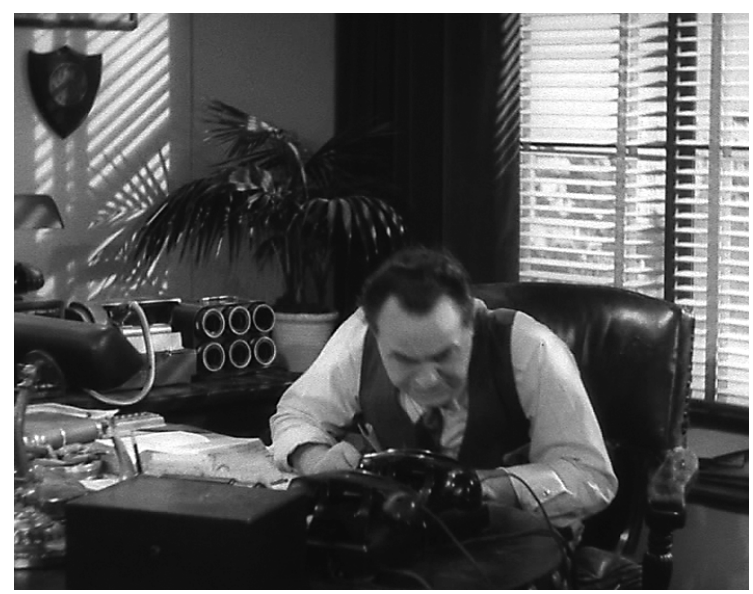

Filmstill aus Double Indemnity, Regie: Billy Wilder, USA 1944
Zweitens dann eröffnet das Motiv Jalousie den Figuren bestimmte Handlungsoptionen. Sie werden nicht nur in das Jalousienlicht, sondern auch unter Handlungsanleitung gesetzt. Erst die Kombination aus Aktanten, aus Licht, Raum, Körper und Jalousie macht überhaupt aus der Figur einen Handlungsträger, einen Akteur. Dessen Optionen und Operationen haben, soweit sie eben vom Motiv der Jalousie induziert sind, markanterweise mit dem Blick zu tun, genauer: mit der Differenz zunächst einmal des Sichtbaren und des Unsichtbaren. Die Jalousie erlaubt den Figuren den Versuch, hinauszusehen (aus dem fiktionalen Innenraum), ohne dabei gesehen zu werden. Sie motiviert also einen bestimmten, filmtypischen Blick (hinaus, hinunter), der ohne sie so nicht möglich wäre und der handlungssetzenden Charakter annehmen kann. Handlungsspielraum eröffnet die Jalousie aber nicht nur den Figuren, sondern auch dem Bild selbst. Eine Jalousie kann verstellt werden (durch wen auch immer: die Filmfigur oder den Szenenbildner), das gerade ist ihr Zweck. Das Lichtmuster des Bildes ist also ein veränderliches, es könnte auch anders werden, ganz hell oder ganz dunkel.

Damit gelangen wir aber schon zu einem dritten Grundzug des Jalousienmotivs. Sehr wichtig ist nämlich, dass die durch die Jalousie motivierte und an die Figur delegierte Blickhandlung zugleich als bildsetzende Operation des Bildes selbst auftritt. Die Ermöglichung nicht nur der Figurenhandlung bzw. eines spezifischen Figurenblicks, sondern auch diejenige der Bildsetzung selbst erscheint im Fall der Jalousie ihrerseits als Motiv im Bild. Dies geschieht, wenn die Grenzen des diegetischen Raumes in der Jalousie umgelegt werden auf die Grenzen des Bildes selbst, also auf die Kadrierung. Die Jalousie wird genau auf die seitliche oder die rückwärtige Grenze des Bildes platziert, wie etwa - ein prominentes Beispiel unter sehr vielen anderen - in der Hotelszene am Anfang von Hitchcocks Psycho. Als sichtbares Objekt verdankt sich das Filmbild eben dieser Operation, die dann mithilfe der Jalousie erneut innerhalb des Bildes als Objekt des Bildes motiviert wird. Gerade dabei zeigt sich auch der offene Charakter des Filmmotivs: die Jalousie eröffnet nämlich einen Spiel- oder Möglichkeitsraum. Immer zeigt sie, anders als beispielsweise die geschlossene Wand, an, dass jenseits ihrer ein weiterer Raum liegt, aus dem das Licht dringt, in das das Bild gesetzt ist und das das Bild als Lichtbild erst möglich macht. Und immer trennt sie, anders als das durchsichtige Fenster, diesen Raum, dessen Vorhandensein sie markiert, dennoch vom Blick ab. Ob sie aber einen Studioscheinwerfer oder die Sonne vom Blick abschirmt, bleibt unentscheidbar. Zusätzlich leistet die Jalousie Ähnliches mit der Unterscheidung des Sehraums vom Hörraum: sie lässt den Off-Ton passieren. In Rita Hayworths berühmter Gesangssequenz in Gilda wird das beson- 
ders deutlich: Neben jedem der beiden jalousienverhängten Fenster, die vom Büro auf den Bühnenraum hinuntergehen, ist ein Abhörgerät installiert, so dass Sehen und Hören getrennt voneinander schaltbar werden, durch das radioartige Gerät der Ton, durch die Jalousie das Bild.

Das Jalousienmotiv kennt auch eine eigene kinematografische Entwicklungsgeschichte; wir können beobachten, wie es sich allmählich herausbildet. Schon bei Griffith etwa feuern die Eingeschlossenen durch die Bretterritzen nach draußen; im Western schaut der Revolverheld durch die Zwischenräume der Planken, mit denen das Fenster vernagelt ist, nach unten auf die Straße. Wir können dann sehen,

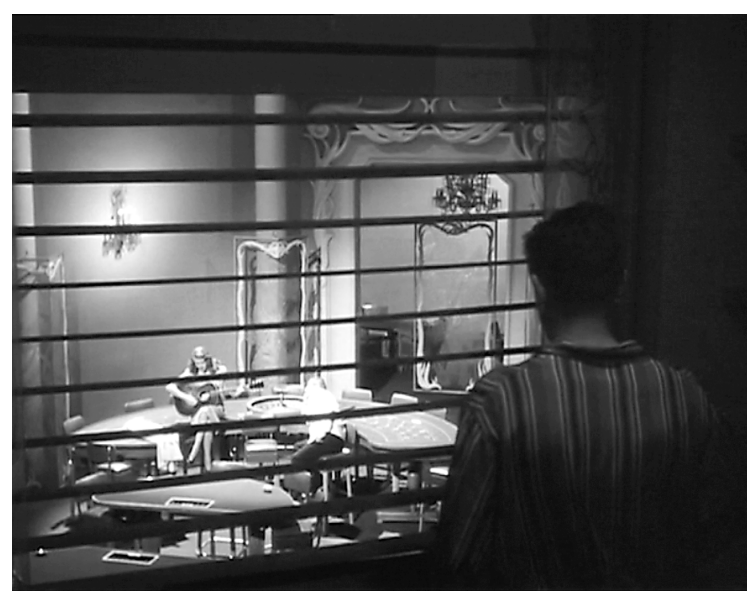

Filmstill aus Gilda, Regie: Charles Vidor, USA 1946 wie die Jalousie in den dreißiger Jahren zunächst akzidentiell als bloßes dinghaftes Requisit - nämlich als Teil der amerikanischen Büroausstattung - auftritt. Sie verdichtet sich zum Motiv, wenn dieses Büro ein Polizei- oder Detektivbüro ist und sie auch geschlossen oder verstellt werden kann. Wir können beobachten, wie dieses Motiv dann in den vierziger Jahren zum Stilelement und sogar zum abrufbaren Versatzstück werden kann. Schließlich fungiert die Jalousie sogar im Wege des pars pro toto zur ikonischen Anzeige ganzer Schauplätze und Milieus oder filmhistorischer Situierungen - man denke an das 〈Neo-Noir $>$ der achtziger und neunziger Jahre.

Und schließlich ist die Jalousie ein gutes Beispiel für ein mediales und intermediales Ausgreifen eines kinematografischen Motivs ins Jenseits des Films. Denn die Jalousie wird in ihrer weiteren Entwicklung vom filmischen zum videografischen oder televisiven Motiv. Sie motiviert dann nicht mehr das kinematografische Bild innerhalb des Bildes, sondern das televisive. Sobald sie nämlich ganz geschlossen wird - wie das im Fernsehfilm der neunziger Jahre auffällig geschieht, besonders im Kriminalfilm -, operiert sie nicht mehr auf der Grenze zwischen innen und außen und mit dem Gegensatz von Licht und Schatten, sondern als hinterleuchtete oder selbst leuchtende Fläche. Sie operiert also als Bildschirm, ohne dabei jedoch die kinematografische Herkunft des Motivs gänzlich abzustreifen - sie kann schließlich jederzeit wieder geöffnet werden und in das Lichtraster-Schema zurückführen. Schließlich greift die Jalousie aber in den realen Raum jenseits des unmittelbar Medialen aus. Sie verwandelt am Ende jeden Raum, in dem sie eingesetzt wird, in einen flexiblen Licht-Raum; sie macht auch außerhalb des Films die Bedingung aller Sichtbarkeit, das Licht, sichtbar anhand des Schattens, der es zwingend begleitet, und auch außerhalb des Films vermittelt sie das Außen- an das Innenlicht und die Zwei- an die Dreidimensionalität. Gerade daher lieben wir ja die Jalousie so sehr, weil sie uns jeden Raum, in dem wir uns bewegen, in ein Licht-Bild, ja ein Leinwandbild verwandelt. Sie erlaubt ein Leben wie im Kino. 
5 Michael Walker, Hitchcock's Motifs, Amsterdam (Amsterdam University Press) 2005.

6 Vgl. dazu Walkers Ausführungen zum Motiv des Schlüssels bei Hitchcock. Ebd., $270 f f$.

7 Vgl. die Motivdiagramme: Ebd., $47 f$.

8 Forschergruppe Telefonkommunikation (Hg.), Telefon und Kultur.

9 Günter Hefler, Libido, diskursiv. Begehren als Motiv im Film, in: Context 21, Nr. 8/2003, Heft 1, 33-37.

10 Thomas Kroll, Im Kino gewesen. Gebetet. Das Nichtalltägliche im Alltäglichen: Das Motiv des Gebets im Film, in: film-dienst, 19/2004, Jg. 57, 44-46.

11 Neben Walker 2005 auch: Philippe Demonsablon, Lexique Mythologique pour l'œuvre de Hitchcock, in: Cahiers du Cinéma 62, August/ September 1956, 18-29, 54-55; Hartmut W. Redottée, Leid-Motive: Das Universum des Alfred Hitchcock, in: Sabine Lenk (Hg.), Obsessionen: Die Alptraumfabrik des Alfred Hitchcock, Marburg (Schüren) 2000, 19-50.

12 Nicole Brenez, Der figurative Unterschied. Pier Paolo Pasolini: II Vangelo Secondo Matteo, in: Christa Blümlinger, Karl Sierek (Hg.), Das Gesicht im Zeitalter des bewegten Bildes, Wien (Sonderzahl) 2002, 163-182.

13 Patrick Primavesi, Tiere in den Filmen von Danièle Huillet und JeanMarie Straub, in: Doris Kern, Sabine Nessel (Hg.), Unerhörte Erfahrung: Texte zum Kino, Frankfurt/M., Basel (Stroemfeld) 2008, 183-208.

14 Hans Jürgen Wulff, Drei Bemerkungen zur Motiv- und Genreanalyse am Beispiel des Gefängnisfilms, in: Jürgen Frieß, S. Lowry, H. J. Wulff (Hg.), 6. Film- und Fernsehwissenschaftliches Kolloquium, Berlin '93, Berlin (Gesellschaft für Theorie und Geschichte audiovisueller Kommunikation) 1994 , 149-154.

15 Michel Cieutat, Les grands thèmes du cinéma américain, Paris 1988-1991.

16 Vgl. Walker, 2005, 17 und David Bordwell, Kristin Thompson, Film Art. An Introduction, 8. Aufl. Boston u. a. (McGraw-Hill) 2008.

17 "One means for fixing important causes or lines of action vividly in our minds is the motif", Kristin Thompson, Storytelling in Film and Television, Cambridge, Mass., London (Harvard University Press) 2003, 25 (Hervorhebung Thompson).

\section{Motivforschung}

Die Jalousie gibt ein Beispiel für das, was unter einem kinematografischen Motiv gefasst werden könnte. Wir nehmen an, dass es daneben sehr viele andere gibt: Hüte, Vasen, Zigaretten, Wind, Regen usw. Jenseits des bloß Beispielhaften aber benötigen wir einen begründeten Motivbegriff. Daher richtet sich der Blick auf bereits vorliegende Arbeiten und Ansätze zur Motivforschung.

Unter den ersten, wenigen kinematografischen Motivuntersuchungen der letzten Zeit sticht besonders Hitchcock's Motifs heraus ${ }^{5}$, das nicht nur ausführlich reflektiert, wieso Motivforschung ein ertragreiches Gebiet sein könnte, sondern das an den Schlüsselmotiven Hitchcocks exemplarisch plausibel macht. Der größte Gewinn von Walkers Versuch, Hitchcocks Werk über seine Motive zu erschließen, liegt vielleicht in der konsequenten Depsychologisierung der Filme. Schnell wird nämlich klar, dass noch die psychologisch aufgeladensten Filme wie etwa Marnie bestimmter Objekte nicht nur als Symbole bedürfen, sondern als Garanten und Anstifter der Funktion eines regelrecht apparativen Systems ${ }^{6}$, welches sich bei genauer Analyse der Motive und ihrer Verbindungen untereinander zum Beispiel als Diagramm darstellen lässt und so bisher ungekannte Relationen sichtbar macht. ${ }^{7}$ Neben diesem jüngsten und ausführlichsten Beitrag zur Motivforschung im Film findet sich eine Reihe von kleineren Arbeiten zu einzelnen filmischen Motiven (etwa dem Telefon ${ }^{8}$, der Libido ${ }^{9}$ oder dem Gebet ${ }^{10}$ ), zu Motiven bei einzelnen Autoren wie Hitchcock ${ }^{11}$, Pasolini ${ }^{12}$ oder Straub-Huillet ${ }^{13}$, in einzelnen Genres ${ }^{14}$ oder einzelnen Nationalkinematografien. ${ }^{15}$

All diese Versuche stehen relativ unverbunden nebeneinander. Sie unternehmen auch kaum Versuche einer genauen Definition des Motivbegriffs sowie einer Bezugnahme auf den Motivbegriff der Literatur-, Musik- und Kunstwissenschaft. Einzig darin stimmen die wenigen Andeutungen überein, dass sie sich meistens auf die narrative und symbolische Funktion des Motivs beziehen ${ }^{16}$ und es als 〈Element〉 des Films von seinen 〈Themen〉 abgrenzen. Das Motiv wird in diesen Lesweisen regelmäßig zum Werkzeug gemacht, mit dem sich, je nach Fokus der entsprechenden Filmwissenschaft, diese oder jene filmische Funktion ableiten lässt. ${ }^{17}$ Für das hier zu skizzierende Projekt scheint es uns dagegen sinnvoll zu sein, das Motiv von dieser Helferfunktion zu befreien und es als eine Instanz eigenen Rechts einzusetzen.

Eher problematisch ist auch eine Bezugnahme auf den in der Literaturwissenschaft entwickelten und hier hoch umstrittenen Motivbegriff ${ }^{18}$ in seiner beachtlichen wie bedenklichen Tradition. ${ }^{19}$ Literarische Motive werden nämlich hier immer schon als Handlungseinheiten aufgefasst, gerade nicht jedoch an auftretende Gegenstände und Dingzusammenhänge gebunden. ${ }^{20}$ Für den Film und das kinematografische Motiv ergibt sich hier das Problem einer Engführung des Films mit dem Narrativen ${ }^{21}$, die Größen wie das Bild, das Licht und die Zeit vernachlässigt. Besonders der Zusammenhang zwischen dem Motiv, der innerdiegetischen 
Handlung und der Handlung des bewegten Bildes selbst oder der Kamerahandlung wird auf diese Weise kaum sichtbar gemacht werden können.

Interessanterweise hebt der Motivbegriff der Musikwissenschaft geradezu auf das Gegenteil ab, nämlich eben nicht auf Handlung und Semantik, sondern auf die Genese der musikalischen Form. ${ }^{22}$ Musikalische Formen wie Rondo oder Sonate folgen jedoch nicht direkt aus einem Motiv, sondern erst aus dem größeren Thema. Insofern ist das Motiv eben nur <formauslösend>, aber noch nicht formkonstitutiv. ${ }^{23}$ Sehr wichtig erscheint aber dabei, dass Vorkommen und Funktionsweise des Motivs sich im Zuge der Entwicklung der Musik verändern: Was ein Motiv ist, wie es arbeitet und was es bewirkt, das lässt sich nur in historischer Relativität angeben. Funktion und Begriff des Motivs in der Musik werden mit dem von der Zweiten Wiener Schule um Alban Berg, Anton von Webern und Arnold Schönberg eingeleiteten Ende der tonalen Harmonik am Beginn des 20. Jahrhunderts gründlich umgestaltet und im Serialismus schließlich gänzlich zurückgenommen. ${ }^{24}$

Für die Entwicklung unseres Motivbegriffs ist die musikwissenschaftliche Motivdiskussion aus zwei Gründen wichtig: Wer aus musikwissenschaftlicher Perspektive nach dem Motiv fragt, der fragt erstens nach einem materiellen, wirksamen und nicht einem semantischen, bedeutsamen Ding. Zweitens sind nicht nur einzelne Motive in ihrem Aufkommen und Verschwinden, sondern in der Weise, in der sie in der Musik grundlegende funktionelle Zusammenhänge stiften, überhaupt historisch wandelbar. Wir vermuten, dass sich eine ähnliche Bewegung auch im Fall des kinematografischen Motivs beobachten lässt.

Um einen Begriff des Motivs in den Kunstwissenschaften hat sich insbesondere Erwin Panofsky bemüht. ${ }^{25}$ Im Vergleich zum literaturwissenschaftlichen Motivbegriff fällt sofort ins Auge, dass das Motiv hier in der konkreten Ansicht konkreter Dinge besteht. $\mathrm{Ob}$ «Tiere, Pflanzen, Häuser, Werkzeuge und so fort» nämlich Handlungen auslösen, sich zu Situationen verdichten oder symbolische Bedeutungen annehmen oder abstoßen, steht auf der Ebene ihrer vorikonografischen Beschreibung (noch) nicht zur Debatte. Mit dem musikwissenschaftlichen Motivbegriff dagegen teilt Panofsky die Bindung des Motivs an das Phänomen der ästhetischen Form, unterscheidet sich aber in der Semantisierung, Trägerschaft «primärer oder natürlicher Bedeutung». ${ }^{26}$

Es lassen sich einige Ergebnisse aus der Beschäftigung mit dem Motivbegriff in Literatur-, Musik- und Kunstwissenschaften für eine kinematografische Motivforschung hier vorläufig festhalten: In der Auseinandersetzung mit der Literaturwissenschaft wird zunächst die Notwendigkeit klar, das Motiv nicht ausschließlich und womöglich nicht einmal in erster Hinsicht als Keim einer erzählten Handlung zu begreifen, sondern es von dieser Einschränkung zu befreien. Am Beispiel der Jalousie konnten wir bereits erkennen, dass das Motiv nicht nur die innerdiegetische Handlung unter Bedingungen setzt oder motiviert, wie den Blick durch die Lamellen, sondern auch an der Bildhandlung, an den bildsetzenden Operationen (wie Kadrierung und Lichtführung) beteiligt ist.
18 Elisabeth Frenzel, Stoff-, Motiv- und Symbolforschung, 4. Aufl. Stuttgart (Metzler) 1978, 31; vgl. auch C. Bremonds Kritik an Stith Thompsons Motif-Index of Folk Literature: Claude Bremond, A critique of the motif, in: Tzvetan Todorov (Hg.), French Literary Theory Today: A Reader, Cambridge, New York u.a. $1988,125-146$.

19 Im deutschsprachigen Raum steht besonders Elisabeth Frenzel mit ihren 'Stoffen der Weltliteratur, und (Motiven der Weltliteratur` für diese Forschungsrichtung. Es darf nicht unerwähnt bleiben, dass Frenzel, die sich durch ihre Rolle im Nationalsozialismus und durch ihre Dissertation zum Judentum im deutschen Theater desavouiert hatte, offenbar nur der Weg in die Zusammenstellung von Stoff- und Motiv-Lexika blieb. "Auf den ersten Blick bietet diese literaturwissenschaftliche Tätigkeit den Vorteil, sich eines eigenen wertenden Standpunktes enthalten zu können.» (Florian Radvan, «... mit der Verjudung des deutschen Theaters ist es nicht so schlimm!m: Ein kritischer Rückblick auf die Karriere der Literaturwissenschaftlerin Elisabeth Frenzel, in: German Life and Letters 54/1, 2001, 25-44, hier 43).

20 Frenzel, Stoff-, Motiv- und Symbolforschung, 29.

21 Wie es beispielsweise einflussreich Christian Metz vertritt.

22 Vgl. Martin Wehnert, Thema und Motiv, in: Die Musik in Geschichte und Gegenwart (MGG1), Bd. 13, Kassel (Bärenreiter) 1966, Sp. 292.

$23 \mathrm{Vgl}$. Hans Mersmann, Angewandte Musikästhetik, Berlin (Max Hesses) 1926, 87.

$24 \mathrm{Vgl}$. Clemens Kühn, Bedeutung von Motiv und Thema in der Musik des 20. Jahrhunderts, in: Musik in Geschichte und Gegenwart, 2. Auflage (MGG2), Sachteil, Bd. 9, Hauptartikel: Thema und Motiv, Sp. 542.

25 Erwin Panofsky, Ikonographie und Ikonologie. Eine Einführung in die Kunst der Renaissance, in: ders., Sinn und Deutung in der bildenden Kunst, Köln (DuMont) 1978, 36-67, hier $38 \mathrm{f}$. 26 Ebd., 40. 
27 Kracauer, Theorie des Films. 28 Vgl. Lorenz Engell, Bilder der Endlichkeit, Weimar (Verlag und Datenbank für Geisteswissenschaften) 2005 .

29 Gilles Deleuze, Le cinéma, tome 1 : L'image-mouvement, tome 2: L'imagetemps, Paris (Minuit) 1982, 1985.

30 Peter Sloterdijk, Du mußt dein Leben ändern, Frankfurt/M. (Suhrkamp) 2009, 56.

31 Zuletzt, überzeugend und prominent: Georges Didi-Huberman, L'image survivante. Histoire de l'art et temps des fantômes selon Aby Warburg, Paris (Minuit) 2002.
Wenn aber das kinematografische Bild vom Motiv in Bewegung gesetzt wird, dann lässt sich allein im Blick auf die erzählten Figuren nicht verstehen, was eine kinematografische Handlung ist. Das filmische Motiv ist deshalb zweitens, anders als das literarische, keine Kern- oder Elementarhandlung, sondern es liegt vor aller Handlung in den audiovisuellen Dingen, sofern sie Beziehungen eingehen mit anderem Sicht- und Hörbarem und darin Handlung produzieren. Damit trägt der kinematografische Motivbegriff vor allem dem schon genannten Umstand Rechnung, dass dem Film schon früh die Besonderheit zugeschrieben wurde, den Fokus vom autonom handelnden menschlichen Subjekt weg und zur Dingwelt hin zu verlagern. ${ }^{27}$ Drittens kommt, das haben wir anhand des musikalischen Motivbegriffs gelernt, das Moment seiner unhintergehbaren Geschichtlichkeit hinzu. Motive verändern sich und ihre Funktion nicht nur, sondern an ihrer Historizität lässt sich womöglich überhaupt verstehen, welche Verhältnisse das Kino zur Geschichte unterhält und welche Zeitform mit dem Kino in die Welt gekommen ist.

Wenn hier von einer Historizität der Motive und ihrer Genealogie und Entwicklung die Rede ist, dann muss jedoch dieser kinematografischen Zeitform eine besondere Nebenbetrachtung gelten. Denn in einem ersten Zugriff könnte man vermuten, dass die Zeitform des Kinos schließlich eine unhintergehbar lineare sei - Filme haben unvermeidlich Anfang, Mitte und Ende. ${ }^{28}$ Dann aber wäre auch ihr Geschichtsverhältnis möglicherweise ein lineares in der Tradition des Historismus. Genau die Linearität der Zeit und der Geschichte ist jedoch spätestens seit dem Ende des iq. Jahrhunderts - zeitgleich mit dem Aufkommen der Kinematografie - mannigfaltig und schließlich massiv in Zweifel gezogen worden. Der Kraft des Films, das lineare, handlungszentrierte Zeitschema abzulösen, hat Gilles Deleuze seine gesamte Filmphilosophie gewidmet. ${ }^{29} \mathrm{Im}$ vorliegenden Zusammenhang ist daran entscheidend, dass diese Zweifel sich durchaus in Auseinandersetzung mit konkreten Dingen und einzelnen Objekten artikulierten und namentlich in den bildenden Künsten greifbar wurden. ${ }^{30}$ Die Entdeckung eines nicht-linearen Zeit- und Geschichtsverhältnisses der bildenden Kunst ist eine der herausragenden Entdeckungen Aby Warburgs, der damit auch die Kunstgeschichte zu einer allgemeinen Kulturwissenschaft hin öffnete. Schlüsselfunktion dabei kommt dem Begriff des <Nachlebens $>$ zu. ${ }^{31}$ Mit ihm fasst Warburg das spontane und diskontinuierliche Auftauchen bestimmter Gesten und Formeln in Kunstwerken quer zu jeder Chronologie und Geografie. Für eine Erforschung kinematografischer Motive ist er doppelt relevant. Zum einen insinuiert der Begriff des «Nachlebens〉, dass die Bilder überhaupt ein eigenes Leben haben, also energetische und kraftvolle Gebilde sind, die jenseits personaler Verhältnisse zu Entwicklung und Veränderung in der Lage sind. Für das bewegte Bild bedarf dieses Argument fast keiner weiteren Ausführungen. Auf die kinematografischen Motive bezogen jedoch bietet der Begriff des 〈Nachlebens $\rangle$ die Möglichkeit, die diskontinuierlichen Austauschverhältnisse zwischen den Bil- 
dern, das, was man auch als das soziale Leben der Bilder bezeichnen könnte, auszuloten. Der energetische Charakter der Bilder ist nämlich nicht immer schon voraussetzungslos verfügbar, sondern bedarf Medien und Orten der Aufführung, um deren Einrichtung und Konzeption sich Warburg zeitlebens bemühte und als die man das Kino ohne Weiteres sehen kann. «Wie Warburgs Atlas die Beziehungen der Bilder auf schwerem, schwarzem Grund eintrug, so verknüpfen filmische Bildatlanten unsere Blicke untereinander und mit der physischen Welt im Dunkel des Kinos.» ${ }^{32}$

Daher ist schließlich für die kinematografische Motivforschung ein vierter Grundzug nach demjenigen der außerdiegetischen Dimension, demjenigen der Dinghaftigkeit und Dinggebundenheit und demjenigen der Historizität ein vierter hinzufügen. Denn wenn die kinematografischen Motive wie Warburgs Gesten und Formeln zwischen den Bildern hin- und herwandern, so verlassen sie den reinen Bildraum und durchqueren die soziale und kulturelle Welt; diese aber ist in medienwissenschaftlicher Sicht immer eine medial konstituierte Welt. Über eine systematische Erforschung von Motiven im Kino ließe sich nicht nur dessen Anschluss an eine allgemeine Geschichte der Bilder (und vermutlich noch darüber hinaus) bewerkstelligen, sondern die verändernde und umwandelnde und also: geschichtliche Kraft des Kinos selbst wäre hier rekonstruierbar, «die Tatsache, daß etwas in der Zeit geschieht, daß Geschichte nicht nur verläuft, sondern verwandelt, daß sie nicht nur aus Daten, sondern aus Ereignissen, nicht nur aus Zwischengliedern, sondern aus Vermittlungen besteht.» ${ }^{33}$ So lässt sich von hier aus die Notwendigkeit einer medien- und sogar kulturwissenschaftlichen Kinoforschung ableiten. Filme sind nicht nur besondere bewegte Bilder, sondern stehen in einem Verhältnis zu anderen Bildern, zu anderen Medien und allen von ihnen ermöglichten sozialen Entitäten wie Menschen, Institutionen oder Wissenschaften. Kinematografische Motive in ihrer Bewegtheit und Bewegung zwischen den Filmen und den Medien artikulieren dieses Verhältnis. Sie weisen uns damit den Weg zu einer Medienwissenschaft als einer «transdisziplinären Kulturwissenschaft des Bildes».34

\section{Motive und Akteure}

Für den hier anvisierten Begriff des kinematografischen Motivs ist mit demjenigen der Zeit das Moment der Bewegung entscheidend. Kinematografische Motive, und das ist das entscheidende Spannungsverhältnis, in dem sie zu begreifen sind, sind einerseits unhintergehbar dinghaft, begegnungsfähige, im Film (möglicherweise auch indirekt: aus dem Off) sicht- und hörbare Objekte. Andererseits sind sie genau so fundamental an Zeit und Bewegung gebunden, an die geschichtliche Bewegung ebenso wie an diejenige des bewegten Bildes oder der handelnden Figuren. Dies gilt so sehr, dass wir sie selbst als handelnde Figuren begreifen wollen: sie veranlassen Bewegungen. Darin genau besteht der Kontrast zur literarischen Tradition der Motivforschung.
32 Ebd., 270.

33 Ebd., 374.

34 Karl Sierek, Foto, Kino und Computer. Aby Warburg als Medientheoretiker, Hamburg (Philo Fine Arts) 2007, 13. 


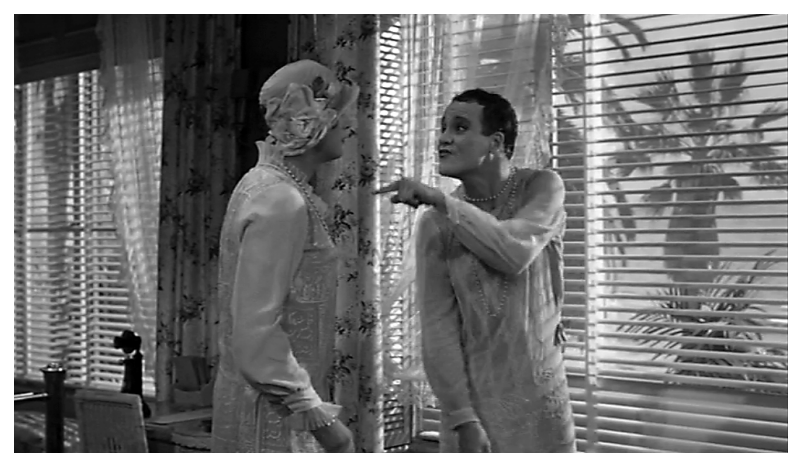

Filmstill aus Some Like it Hot, Regie: Billy Wilder, USA 1959
35 Bruno Latour, Eine neue Soziologie für eine neue Gesellschaft. Einführung in die Akteur-Netzwerk-Theorie, Frankfurt/M. (Suhrkamp) 2007, 123.
Bewegungen zu veranlassen heißt, Unterschiede zu bewirken. «[J]edes Ding, das eine gegebene Situation verändert, indem es einen Unterschied macht, [ist] ein Akteur - oder, wenn es noch keine Figuration hat, ein Aktant.» ${ }^{35}$ Was Latour als Akteur bezeichnet, wollen wir als Motiv bezeichnen, während der Aktant ein bloßes, einfaches Ding im Film wäre. Ein solches einfaches Ding im Film könnte beispielsweise ein silbrig glänzendes kleines Stück Metall sein, das irgendwo in einem Filmset auf einem Garderobenschrank liegt und das wir vielleicht, wenn wir Glück haben und die Einstellung nah genug ist oder sich die Kamera langsam genug bewegt, als Schlüssel identifizieren können. Filme sind voll solcher vorhandener, aber funktionsloser Dinge: eine Vase auf einem Fensterstock, eine männliche Figur, die im Hintergrund durch das Bild geht. Diese Dinge könnten möglicherweise einmal an einer Handlung beteiligt sein. So lange sie es nicht sind, fehlt ihnen die Figuration. Sie sind nur irgendeine männliche Figur und irgendeine Vase und nicht der Mörder, der mit der Vase jemanden töten wird. Das Zusammentreffen der einfachen Dinge im Raum und im Licht und in der Bewegung des Films erst macht sie zu Akteuren, die nicht nur auf der Ebene des Plots etwas tun (nämlich einen Mord begehen), sondern auch eine bestimmte Art der Montage ermöglichen: ohne Revolver keine amerikanische Einstellung. Diese Figuration, die aus einem Mann und einer Vase einen Mörder macht, entscheidet darüber, was ein Motiv und was nur ein einfaches Ding im Film ist.

Für eine Motivforschung würde es also nicht genügen, einfach nur alle Vasen der Filmgeschichte zu sammeln, sondern man müsste angeben, wann und unter welchen Bedingungen Vasen bei der Konstitution von Mördern im Spiel sind, warum manche Vasen einfach nur Vasen bleiben, und warum andere wiederum über das Schicksal der verlassenen Filmheldin entscheiden (der Unterschied zwischen einer Vase mit weißen Lilien und einer Vase mit roten Rosen). Und man müsste angeben können, welche filmischen Operationen etwa in Montage, Kadrage, Licht- und Tonführung dabei anfallen. Denn das kinematografische Motiv ist das konstituierende Moment für wirkliche, tatsächlich durchgeführte Operationen des Bildes und nicht nur für imaginierte Plot-Handlungen; es interessiert sich nicht für die theoretische Trennung von Diegese und Nicht-Diegese. Nur in einem spezifischen Arrangement aus Kamera, Kadrierung, Licht, Objekten, Studiostrukturen, Figuren, Ton, Text ergibt sich etwas, das später als Handlung erkennbar gewesen sein wird. Insofern ist im kinematografischen Motiv also eine wirkliche und wirksame Kraft zu sehen und die Handlungen, die sich am Leitfaden von Kino-Motiven beschreiben lassen, sind keine imaginierten oder repräsentierten, sondern wirkliche Handlungen eines wirklichen Kinos. 


\section{Die Motivdatenbank}

Kinematografische Motivforschung im hier angestrebten Sinn steht in der Gefahr, zu viele Kräfte für das Sammeln der Motive aufzehren zu müssen, statt sie für die eigentliche Arbeit an den Motiven einsetzen zu können. Wir schlagen deshalb vor, dieses Problem durch die Einrichtung einer frei verfügbaren und weltweit zugänglichen elektronischen Motivdatenbank zu lösen. ${ }^{36}$ In diese Datenbank könnten alle Interessierten sowohl Motivfunde einpflegen als auch aus ihr Abfragen generieren.

Es hat im Bezug auf Warburg und in den Bildwissenschaften bereits Versuche gegeben, solche Systeme zu erstellen: etwa die Warburg Electronic Library (WEL), um nur die wichtigste zu nennen. Projekte wie dieses haben sehr ermutigende Ergebnisse zu Tage gefördert, mussten sich jedoch alle mit dem schwierigen Problem der Schlagwortvergabe und der Zugriffshierarchien befassen. ${ }^{37}$ Im Vergleich dazu steht die kinematografische Motivforschung jedoch vor einer anderen Ausgangslage: Es gibt keine Originale von Filmen wie von Tafelbildern, sondern im Regelfall nur zirkulierende Kopien. Filme, die auf Medien wie VHS, DVD oder neuerdings Blueray zirkulieren, haben einen noch größeren Verbreitungsgrad. Die bewegten Bilder sind also schon immer in einem höheren Maße verteilt als die unbeweglichen. Schon heute stehen in frei verfügbaren Datenbanken wie Youtube oder der $I M D B$ (bewegte) Bilder in kaum noch zu überschauendem Umfang bereit. Die hier vorgeschlagene Motivdatenbank will diese Verteilung und Offenheit nutzen. Sie strebt eine möglichst große Vernetzung mit derartigen Datenbanken an. ${ }^{38} \mathrm{Um}$ das volle Potential einer weltweit vernetzen Gemeinde von Motivsuchern auszuschöpfen, schlagen wir deshalb vor, ein solches System mit selbstregulativen Fähigkeiten auszustatten, wie sie in den letzten Jahren im Kontext von sozialen Netzwerken wie Facebook, aber auch bereits in Wissensdatenbanken wie der Wikipedia oder der $I M D B$ erprobt wurden. ${ }^{39}$

Es ist allerdings ein zweites Forschungsdesiderat für die Entwicklung der Motivdatenbank, auch für die Auswertung technische und methodische Lösungsvorschläge zu unterbreiten. Man könnte sich etwa vorstellen, ein Abfragemodul für interessierte Laien zur Verfügung zu stellen, das völlig eigene Ordnungssysteme ermöglicht, und ein anderes System für filmwissenschaftliche Forschungen, welches wissenschaftsintern anerkannte Kategorien bereits mit implementiert. Durch die Anordnung in relationalen Datenbanken lässt sich so etwas bewerkstelligen: im Datenbestand von Ebay ist es ebenso möglich, assoziativ durch Produktvorschläge zu navigieren wie sich entlang streng hierarchischer Kategorienbäume vorzuarbeiten: Der Datenbestand selbst bleibt davon unberührt. Spätestens die Arbeit an einem populären Medium wie dem Kino darf die Begegnung mit nicht im strengen Sinn wissenschaftlicher Wissensproduktion daher nicht nur nicht scheuen, sondern muss sie geradezu suchen.

36 Wir werden uns in absehbarer Zeit um die Einrichtung einer entsprechenden Forschergruppe bemühen.

37 Matthias Bruhn, Der Bilderatlas vor und nach dem Zeitalter der Digitalisierung, in: Sabine Flach, Inge Münz-Koenen, Marianne Streisand (Hg.), Der Bilderatlas im Wechsel der Künste und Medien, München (Fink) 2005, 181-197, hier 192.

38 Ebd., 197.

39 Von bereits bestehenden Projekten kommt dem am nächsten die Internet Movie Car Database IMCDb. 


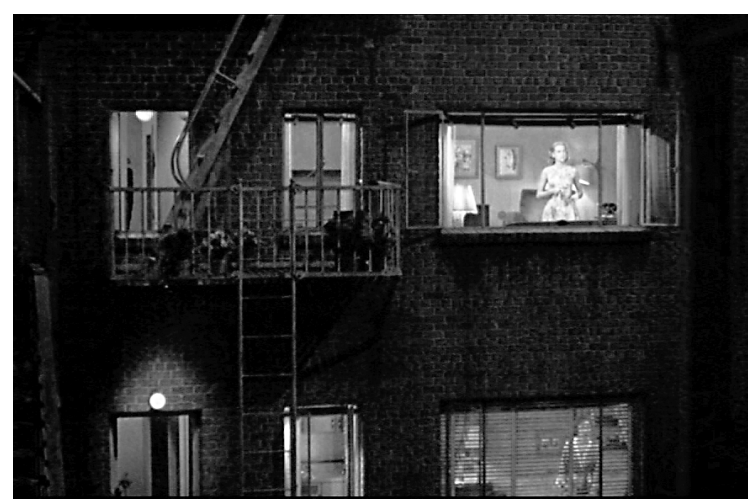

Filmstill aus Rear Window, Regie: Alfred Hitchcock, USA 1954

\section{Die Forschungskonsequenzen}

Was wäre nun der denkbare Ertrag einer kinematografischen Motivforschung für die Filmwissenschaft? Sie sind derart reichhaltig, dass hier zunächst eine ganz kurze, erste Skizze genügen muss. ${ }^{40}$

Der erste zu erwartende Effekt wäre die Dynamisierung eines ganzen Sets von Begriffen der Filmforschung und der filmischen Lektürepraxis. Filme würden quer zu Genres, quer zu Autoren, quer zu Stilen und Epochen lesbar werden. Genres etwa ließen sich wohlmöglich als gemischte Ensembles beschreiben, die durch ein Zusammenwirken kinematografischer Motive einerseits überhaupt erst entstehen, ihnen andererseits einen Schauplatz geben. Damit ist angedeutet, dass sich Konsequenzen auch für die Filmgeschichtsforschung ergäben. Wenn Genres und dann möglicherweise auch Stile, Epochen, Autoren als Produkte einer Interaktion der Motive verfügbar sind, dann sind sie zugleich auch als die Reproduktionsbedingungen der Motive kenntlich gemacht. An die Seite einer Geschichte des Films müsste folglich die Evolution der Motive treten; zur kinematografischen Historiografie eine Genealogie; ja sogar, wie gesehen, die Erforschung des diskontinuierlichen warburgschen «Nachlebens〉 im Film.

Schließlich und entscheidend betrachtet kinematografische Motivforschung Filme quer zu Filmen, ja sogar quer zum Film als Medium. Genau hier öffnet sich dann auch das Feld vom Film über den Film hinaus auf eine allgemeine Medialitätsund Bilderforschung. Sie würde mindestens in dreierlei Blickrichtung verlaufen. Zum ersten wäre nämlich zu untersuchen, wie Filmmotive die Grenzen des Films auflösen (ohne dabei den Film aufzulösen). Viele filmische Motive, so auch die hier erwähnten Beispiele, haben ihre Herkunft vermutlich gar nicht im Film, sondern in der Architektur, dem Design, der Werbung, im Handwerk oder der technisch-industriellen Dimension des Alltags. Und sie dringen, jenseits des Films, in die Welten etwa der Computerspiele und des Webdesigns, aber auch zurück in ihre Herkunftsdimensionen ein. Sie bleiben dabei filmische Motive, begegnen aber außerhalb des Films, und werden schließlich vielleicht sogar als filmische durch Adaption an andere Kontexte unkenntlich.

In einer zweiten Richtung wäre die hier vorgeschlagene kinematografische Motivforschung so lange unvollständig, wie sie nicht von einer medienwissenschaftlichen (und metawissenschaftlichen) Begleitforschung ihrerseits beobachtet würde. Auf einem wohldefinierten Feld könnte einmal begleitend beobachtet werden, wie ein materielles Werkzeug (die Motivdatenbank) und ein immaterielles Werkzeug (das hier skizzierte Konzept des Filmmotivs) an der Genese eines Wissensobjekts und damit an der Urheberschaft der Erkenntnisse mitwirken. Das würde die Filmforschung zwar im Ganzen zunächst zum Objekt einer zweiten Beobachtungsebene
$40 \mathrm{Vgl}$. dazu auch Sierek, Foto, Kino und Computer, $24 \mathrm{ff}$. 
herabsetzen, aber andererseits ihre Anschlussfähigkeit an aktuelle kulturwissenschaftliche und wissenssoziologische Debatten sicherstellen.

Und schließlich und drittens wäre, derart anschlussfähig geworden, der Blick gänzlich auf andere Medien zu richten. Filme sind für die kinematografische Motivforscbung beschreibbar als je spezifische Agenturen, Zusammensetzungen und Zusammenwirkungen heterogener Motive. Wenn aber solche Motive sich auch außerhalb des Films zusammenballen und in andere, nicht-kinematografische oder gar gemischte, intermediale Agenturen eingehen - und auch aus der Intermedialitätsforschung wissen wir, dass jedes einzelne Medium ein Intermedium ist, das die anderen Medien konfiguriert, reflektiert, transformiert $-{ }^{41}$, dann sind auch andere Medien möglicherweise über die Zusammenwirkung bestimmbarer und materiell gegründeter Motive zu beschreiben.
41 Jens Schröter, Intermedialität, http:||www.theorie-der-medien.de| text_detail.php?nr=12, gesehen am 29.3.2009; dort auch ein ausführliches Literaturverzeichnis. 